\title{
Particle Swarm Optimization Based Beamforming in Massive MIMO Systems
}

\author{
https://doi.org/10.3991/ijim.v14i05.13701
}

\author{
Thaar A.Kareem $\left.{ }^{(}\right)$, Maab Alaa Hussain, Mays Kareem Jabbar \\ University of Misan, Misan, Iraq \\ thaar kareem@uomisan.edu.iq
}

\begin{abstract}
This research puts forth an optimization- based analog beamforming scheme for millimeter-wave (mmWave) massive MIMO systems. Main aim is to optimize the combination of analog precoder / combiner matrices for the purpose of getting near-optimal performance. Codebook-based analog beamforming with transmit precoding and receive combining serves the purpose of compensating the severe attenuation of mmWave signals. The existing and traditional beamforming schemes involve a complex search for the best pair of analog precoder / combiner matrices from predefined codebooks. In this research, we have solved this problem by using Particle Swarm Optimization (PSO) to find the best combination of precoder / combiner matrices among all possible pairs with the objective of achieving near-optimal performance with regard to maximum achievable rate. Experiments prove the robustness of the proposed approach in comparison to the benchmarks considered.
\end{abstract}

Keywords - Millimeter-wave, Beamforming, Massive MIMO, PSO optimization

\section{Introduction}

The ever-increasing number of users and the demand for improved quality of service requires the development of new and better standards in wireless telecommunication. The new $5^{\text {th }}$ generation systems $(5 \mathrm{G})$ appear to be a promising step in such a direction that can reliably meet the ever-increasing consumer demand. Next Generation Mobile Networks Alliance $[1,2]$ has put forth the following list of requirements for $5 \mathrm{G}$ :

- Increased data rates

- Decreased latency rate

- Ability to handle large number of connected devices

- Increased dependability.

As compared to $4 \mathrm{G}$, the recent $5 \mathrm{G}$ networks claim to have the ability to increase capacity by 1000 times before 2020 [2, 3]. There are many methods that can increase capacity; for example, by increasing spectral efficiency using physical layer (massive multiple-input-multiple-output (MIMO) and advanced channel coding [4, 5]). Area spectral efficiency can be increased by deploying Network densification that includes: 
- Permitting device-to- device (D2D) communications [6]

- By deploying small cells $[7,8]$

- By enabling advanced cooperation, such as Cloud-RANs $[9,10]$.

It is not possible to increase capacity of the existing telecommunication systems further because of the inherent restriction constraints. Utilization of the hereby underutilized frequency spectrums thus looks like a promising way out of this predicament [3].

The hereby underutilized spectrum of millimeter wave (mmWave) frequency band is now being considered for the very purpose to design broadband cellular communication networks of the future [11]. The frequency range used by it is within $30 \mathrm{GHz}$ to $300 \mathrm{GHz}$. Present between microwave and infrared spectrum range, this spectrum can be used for efficient wireless communications. This band until recently was only used as a medium to transmit indoor high-resolution multimedia streams [12] and for open-air point-to-point backhaul links [13]. Recent research has now shed light on the suitability of mmWave for open-air telecommunication [11, 14].

The main reason behind the growing interest in mmWave spectrum is that the amount of available bandwidth is gigantic as compared to that of $4 \mathrm{G}$ and its predecessor wireless telecommunication frameworks.

Its carrier frequency is ten times more than its predecessors, making its wavelength subsequently smaller. On one hand it is a plus-point because:

- It enables mmWave precoding to combat large scale transceiver antennas for providing large beamforming gains to counter path loss and to create highly directional beams

- They exploit polarization well along with other new spatial processing techniques, such as adaptive beamforming and massive MIMO [15]

On the other hand this very characteristic of smaller wavelength poses a challenge to its adaptability because of the huge path loss and rain attenuation that results from it [14].

In traditional MIMO systems, all antenna elements are linked to a baseband processor. This calls for the use of a dedicated mixer, analog to digital converter (ADC) / digital to analog converter (DAC), filters and amplifiers per antenna. The series of elements that link an antenna to baseband are called radio frequency (RF) chains. Digital precoders in traditional MIMO systems are located at baseband. They adjust signal magnitude as well as signal phase. Directional signal transmission and reception is achieved by the technique of beamforming. Beamforming fundamentally steers the signal in a particular direction. Its elemental principle is to modify the amplitude and phase for power variation and beam steering. Different antenna arrays with different provision and arrangement for amplitude and phase variation are used in beamforming for transmission and reception. 

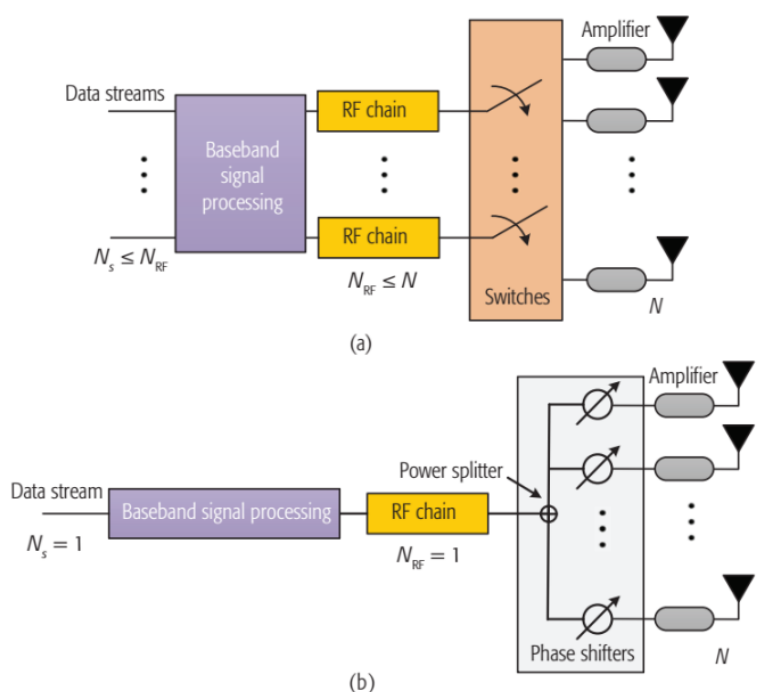

Fig. 1. Architectures of traditional low RF-complexity technologies: a) antenna selection; b) analog beamforming [16]

In fully digital precoders, analogue to digital converters (ADCs) and radio frequency (RF) chains should be same in number as the total antenna elements.

The large frequencies of mmWave channel permit using large number of antennas but with the increase in antenna elements, the number and cost of RF chains would also increase, thus posing a constraint, rendering digital precoding infeasible in mmWave spectrum.

A recently proposed hybrid precoding model on the other hand requires only a small number of RF chains interfaced between a low dimensional digital pre coder and a high dimensional analog pre coder [17]. High dimensionality of analog pre coders makes it infeasible to be used in RF domain where it is required to use mega power variable voltage amplifiers (VGAs) [10].

This calls for a trade-off between low cost phase shifters at analog pre coder side and the reduced ability to adjust the magnitude of RF signals.

MmWave massive MIMO systems typically perform analog beamforming. Analog phase shifter (PS) network is used to change phase and has quite low hardware cost [14, $18,19]$. There are two main types of analog beamforming schemes:

- Non-codebook-based beamforming

- Codebook-based beamforming

Non-codebook beamforming methods have the ability to get superior optimal performance but they need exact channel state information (CSI) at Base Station (BS)which is not feasible when the number of expensive RF chains is kept low [10]. On the contrary, codebook-based beamforming can function even without perfect CSI information. It involves predefined codebooks of all possible analog precoder and 
combiner matrices and the algorithm searches for such a pair that gives best performance. Only downside is that this technique of beamforming incurs a large computational cost that occurs when iteratively exchanging information between BS and user to search for the optimal pair. Also, with the increase in RF chains and quantified bits of the angles of arrival and departure (AoA/AoDs), complexity rises [20].

[21]in 2015 proposed a Turbo-TS analog beamforming approach that performs this search for best precoder and analog pair by using machine learning, i-e optimization. This eliminates the need for checking all possible combinations from the codebooks, hence reducing complexity. Their main approach is made up of two parts:

- A Turbo-like joint search scheme based on continuous information exchange between user and BS

- TS-based precoding/combining that uses local search to find the best precoder and combiner pair from among the predefined codebooks.

Their simulation results proved that the complexity of this approach is linear with number of RF chains, and does not depend on quantified bits per angles of arrival and angles of departure.

In this paper, we propose the use of Particle Swarm Optimization to perform this search.

\section{System Model}

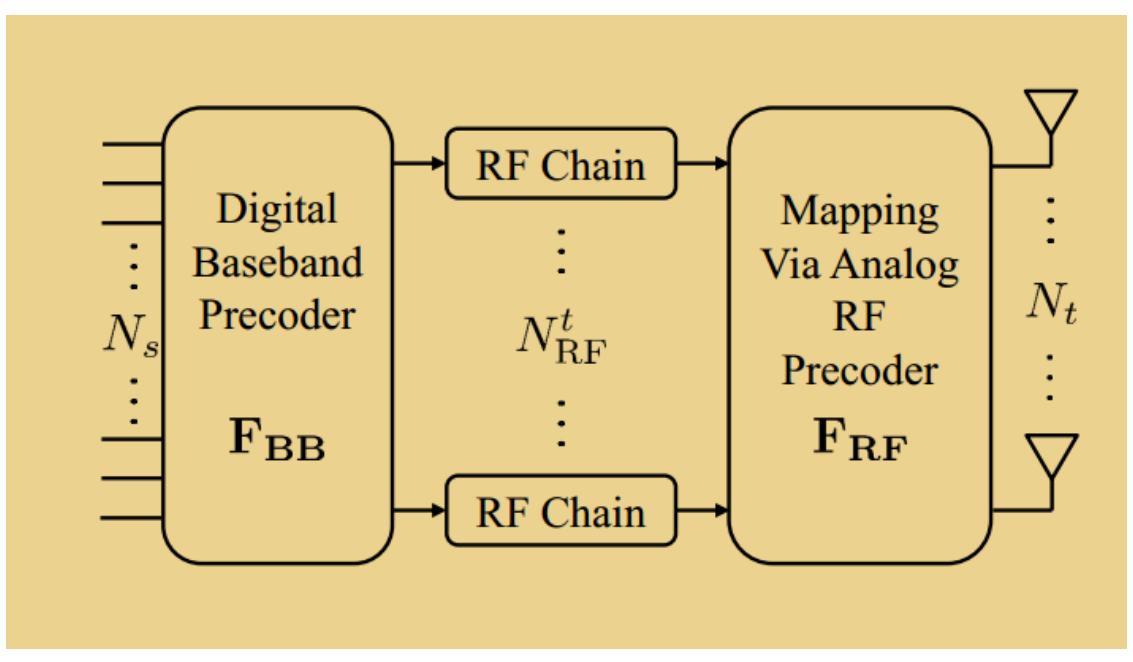

Fig. 2. MmWave MIMO transmitter architecture [18]

Consider a mmWave massive MIMO architecture as shown below: 


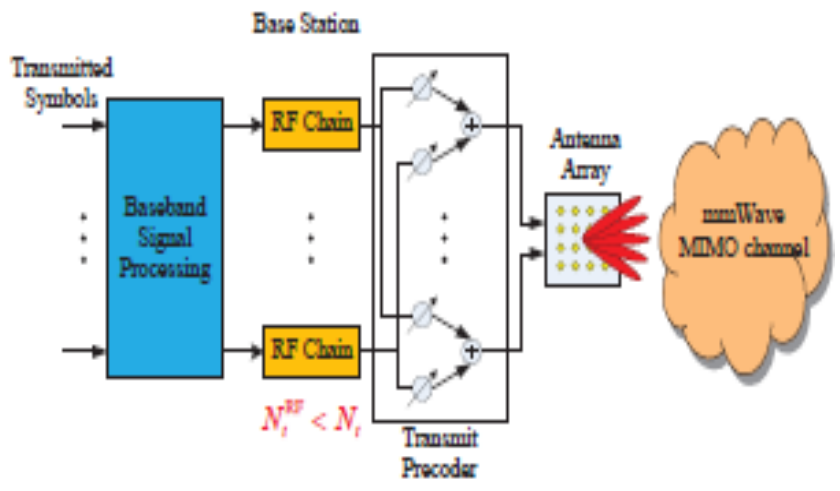

(a)

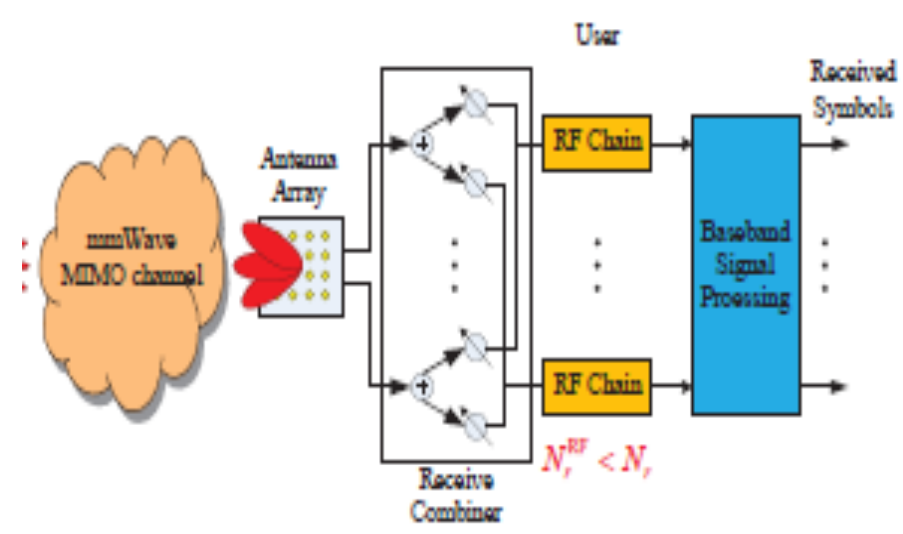

(b)

Fig. 3. (a) Beamforming of Massive MIMO at Transmitting end; (b) Beamforming of Massive MIMO at Receiving end

A total of $N_{t}$ antennas are installed at base station in conjunction with $N_{t}^{R F}$ RF chains.

$N_{s}$ antennas are used at the transmission end, by which $N_{s}$ data streams are transmitted to a user having $N_{s}$ antennas on the receiver end.

Maximum spatial multiplexing gain is achieved by setting:

$$
N_{t}^{R F}=N_{r}^{R F}=N_{s}
$$


The overall procedure goes like this: $N_{s}$ data streams pass through $N_{t}^{R F}$ RF chains after which they are converted to analog signals. These signals are precoded by an $N_{t} \times N_{t}^{R F}$ analog precoder, $P_{A}$, after which they are transmitted.

$$
\mathrm{x}=P_{A} S
$$

Here, $\mathrm{s}$ is the $N_{s} \times 1$ transmitted data which has normalized power $E\left(\mathrm{ss}^{H}\right)=\frac{1}{N_{s}} I_{N_{s}}$

PA is based on a PS network with low hardware complexity [22] and all its elements satisfy the condition

$$
\left|p_{i, j}^{A}\right|^{2}=\frac{1}{N_{t}}
$$

The signal vector received at the user side is of dimension $N_{r} \times 1$. If $\rho$ denotes the total transmitted power and $\mathrm{H} \in \mathbb{C}^{N_{r} \times N_{t}}$ represents the channel matrix then with narrowband block-fading massive MIMO channel [16], the received signal, $r$ can be modelled as

$$
r=\sqrt{\rho} H P_{A} s+n
$$

where $\mathrm{n}=[\mathrm{n} 1, \cdots, \mathrm{nNr}]^{T}$ is the additive white Gaussian noise (AWGN), whose values are based on the identical and independent distribution (i.i.d.) $C N\left(0, \sigma^{2} \mathrm{I}_{N_{r}}\right)$.

The received signal $\mathrm{r}$ at the user end is processed by an $N_{r} \times N_{r}^{R F}$ analog combiner $C_{A}$

$$
y=C_{A}^{H} r=\sqrt{\rho} C_{A}^{H} H P_{A} s+C_{A}^{H} n
$$

where $C_{A}$ elements have constraints similar to that of $P_{A}$. 


\section{$3 \quad$ Methodology}

The overview of methodology adopted to implement the proposed method is shown in the figure below.

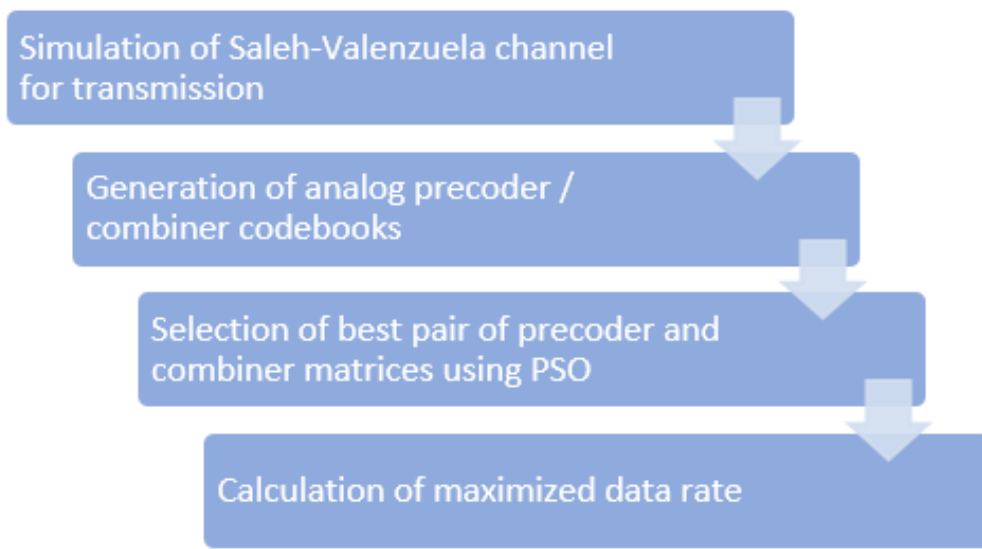

Fig. 4. Overall algorithm flow

\subsection{Channel modelling}

First step in the process is the modelling of the channel. We base our simulations on a geometric channel model with very limited number of significant scatters because of the existence of heavy antenna correlation in mmWave systems [23] SalehValenzuela channel [24] which is a widely popular channel model, was thus chosen. It is basically an extension of Turin model.

It statistically explains the clustered property of calculated received power delay profiles in indoor setting.

The Saleh and Valenzuela channel matrix $\mathrm{H}$ can be modelled by the following equation:

$$
H=\sqrt{\frac{N_{t} N_{r}}{L}} \sum_{l=1}^{L} \alpha_{l} f_{r}\left(\phi_{l}^{r}\right) \mathrm{f}_{t}^{H}\left(\phi_{l}^{t}\right)
$$

Here the number of significant scatters is represented by L. in mmWave spectrum due to the sparse nature of scatters we have $L \leq \min \left(\mathrm{N}_{t}, \mathrm{~N}_{r}\right)$.

lth path gain is $\alpha_{l} \in \mathbb{C}$. It includes path loss.

Azimuth of AoDs/AoAs of the lth path are represented by $\phi_{l}^{t}$ and $\phi_{l}^{r}$ respectively. 


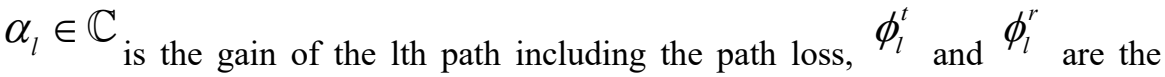
azimuth of AoDs/AoAs of the lth path, respectively.

Antenna array response vectors are given by $f_{t}\left(\phi_{l}^{t}\right)$ and $f_{r}\left(\phi_{l}^{r}\right)$ respectively. These are governed by the antenna array structure of BS and user.

For uniform linear arrays (ULAs) considered in this research:

$$
\begin{aligned}
& f_{t}\left(\phi_{l}^{t}\right)=\frac{1}{\sqrt{N_{t}}}\left[1, e^{j k d \sin \left(\phi_{l}^{t}\right)}, \cdots, e^{j\left(\mathrm{~N}_{t}-1\right) k d \sin \left(\phi_{l}^{t}\right)}\right]^{T} \\
& f_{r}\left(\phi_{l}^{r}\right)=\frac{1}{\sqrt{N_{r}}}\left[1, e^{j k d \sin \left(\phi_{l}^{r}\right)}, \cdots, e^{j\left(\mathrm{~N}_{r}-1\right) k d \sin \left(\phi_{l}^{r}\right)}\right]^{T}
\end{aligned}
$$

If wavelength of the signal is given by $\lambda$ and antenna spacing is given by $d$, then

$$
k=\frac{2 \pi}{\lambda}
$$

\subsection{Generation of codebooks}

Each beam steering codebook [25] of mmWave channel consists of a matrix whose columns represent beamforming weight vectors. These contain information regarding beam direction. Each codebook has matrices such that the total beam patterns in the codebook are generated symmetrically throughout the 360 degrees around the antenna device. This ensures that the gain loss at the overlap of any two successive patterns is as low as possible.

Suppose that the beam steering codebook of analog precoder is represented by F. It enlists all feasible analog pre coder matrices, represented by $P_{A}$.

Also suppose that the beam steering codebook of analog combiner is represented by W. It enlists all feasible analog combiner matrices, represented by $C_{A}$.

$$
\begin{aligned}
& P_{A}=\left[f_{t}\left(\bar{\phi}_{1}^{t}\right), f_{t}\left(\bar{\phi}_{2}^{t}\right), \cdots, f_{t}\left(\bar{\phi}_{N_{t}^{R F}}^{t}\right)\right] \\
& C_{A}=\left[f_{r}\left(\bar{\phi}_{1}^{r}\right), f_{r}\left(\bar{\phi}_{2}^{r}\right), \cdots, f_{r}\left(\bar{\phi}_{N_{r}^{R F}}^{r}\right)\right]
\end{aligned}
$$


If the number of bits to calculate AoD (AoA) is represented by $B_{t}^{R F}\left(B_{r}^{R F}\right)$, then each AoD $\bar{\phi}_{i}^{t}$ will range from $\mathrm{i}=1$ to $\mathrm{i}=N_{t}^{R F}$. So each computed AoD will have $2^{B_{t}^{R F}}$ possible values.

$$
\bar{\phi}_{i}^{t}=\frac{2 \pi n}{2^{B_{t}^{R F}}} \text { where } \mathrm{n} \in\left\{1, \cdots 2^{B_{t}^{R F}}\right\}
$$

Cardinality $|\mathrm{F}|$ of $\mathrm{F}$ will therefore be $2^{B_{t}^{R F} \cdot N_{t}^{R F}}$.

Also, each AoA, $\bar{\phi}_{j}^{r}$ will range from $\mathrm{j}=1$ to $\mathrm{j}=N_{r}^{R F}$. So, each AoA will have $2^{B_{r}^{R F}}$ possible values.

$$
\bar{\phi}_{j}^{r}=\frac{2 \pi n}{2^{B_{r}^{R F}}} \text { where } \mathrm{n} \in\left\{1, \cdots 2^{B_{r}^{R F}}\right\}
$$

Cardinality $|\mathrm{W}|$ of $\mathrm{W}$ will therefore be $2^{B_{r}^{R F} \cdot N_{r}^{R F}}$.

\subsection{Definition of cost function}

Each possible combination of precoder and combiner matrix will give a different achievable rate. We aim to search for such a pair that will maximize the achievable rate.

The maximum achievable rate given by any combination of precoder $P_{A}$ and combiner $C_{A}$ can be given as

$$
\begin{gathered}
R=\max _{P_{A} \in F, C_{A} \in W} \log _{2}\left(\left|I_{N_{s}}+\frac{\rho}{N_{s}} R_{n}^{-1} C_{A}^{H} H P_{A} P_{A}^{H} H^{H} C_{A}\right|\right) \\
R=\max _{P_{A} \in F, C_{A} \in W} \log _{2}\left(\varphi\left(P_{A}, C_{A}\right)\right)
\end{gathered}
$$

Here

$R_{n}=\sigma^{2} C_{A}^{H} C_{A}$,is the covariance matrix of noise after combining. Hence the above equation can be expressed as:

$$
\varphi\left(P_{A}, C_{A}\right)=\left|I_{N_{s}}+\frac{\rho}{N_{s}} R_{n}^{-1} C_{A}^{H} H P_{A} P_{A}^{H} H^{H} C_{A}\right|
$$


In the next section is described the optimization algorithm that is used to maximize the achievable rate.

\subsection{Particle Swarm Optimization (PSO) optimized selection of pre coder and combiner matrices}

To maximize the achievable rate, the finest combination of pre coder / combiner must be chosen from all possible combinations that were generated using equations 7 and 8. For this objective, PSO is used. In this section we will give an overview of PSO and its mathematical formulation.

PSO is a population-based metaheuristic that prevents a solution from being trapped to local optimum $[26,27]$ and can yield acceptable solutions in a reasonably practical time [28]. It is a popular method to solve different types of optimization problems related to engineering [13]. A potential solution is called a 'particle'. Objective function gives the 'fitness' value of each particle. Particles are initialized randomly. Each particle moves in multidimensional search space with random velocities in search of a better fitness value. This update in the position of a particle takes place based on the information of its personal/ local best position and global best position. This process is repeated in each iteration. Inertia weights help update velocity. Boundary constraints are imposed on the particle to enforce that the particle solution stays within allowed range.

Each candidate solution (or PSO 'particle') here is a possible pair of pre coder $P_{A}$ and combiner $C_{A}$. Each particle can be represented by a point in $\mathrm{D}$ dimensional space, where $\mathrm{D}$ is the number of variables to optimize- which in our case is 2 : first is the index from pre coder codebook (each index has a different $P_{A}$ matrix) and second one is the index from combiner codebook (each index has a different $C_{A}$ matrix). Any single combination of these indices can be mapped to a possible combination of pre coder and combiner matrices.

In D dimensional space, a particle i can be represented as [29]:

$$
x_{i}=\left[x_{i 1}, x_{i 2}, x_{i 3} \ldots \ldots \ldots \ldots, x_{i D}\right]
$$

Each swarm X consists of a population of $\mathrm{N}$ particles.

$$
X=\left[x_{1}, x_{2}, x_{3} \ldots \ldots \ldots \ldots, x_{N}\right]
$$

Particles of a population move in the search space by using the following equation to update their positions.

$$
x_{i}(t+1)=x_{i}(t)+v_{i}(t+1)
$$

In the above equation, $\mathrm{t}$ and $\mathrm{t}+1$ are two consecutive iterations. $\mathrm{Vi}$ is a velocity vector of ith particle along $\mathrm{D}$ dimensions. It controls how the particle moves in the search space. Velocity vector is made up of three components defined below: 
- Inertia/ momentum-which forbids a particle from sharply changing direction.

- Cognitive component c1-which forbids a particle from returning to its previous best position.

- Social component $\mathbf{c 2}$-which ascertains the tendency of a particle to move towards best position of entire swarm.

These components can be represented by the equation:

$$
v_{i}(t+1)=v_{i}(t)+c_{i}\left(p_{i}-x_{i}(t)\right) R_{i}+c_{2}\left(g-x_{i}(t)\right) R_{2}
$$

Here, pi is the local best of a particle and $\mathrm{g}$ is the global best and $0 \leq(\mathrm{c} 1, \mathrm{c} 2) \leq 4$. $\mathrm{R} 1$ and $\mathrm{R} 2$ are diagonal matrices of random numbers generated from uniform distribution in $[0,1]$, so that both $\mathrm{c} 1$ and $\mathrm{c} 2$ randomly affect the velocity update. The same process is repeated for all iterations.

\section{Initialization. For each of the $N$ particles:}

a. Initialize the position $\mathbf{x}_{(}(0) \vee i \in 1: \mathrm{N}$

b. Initialize the particle's best position to its initial position $\mathbf{p}(0)=\mathbf{x}_{f}(0)$

c. Calculate the fitness of each particle and if $f\left(\mathbf{x}_{(}(0)\right) \geq f\left(\mathbf{x}_{(}(0)\right)$ vi $\neq j$ initialize the global best as $\mathbf{g}-\mathbf{x}_{j}(0)$

2. Until a stopping criterion is met, repeat the following steps:

a. Update the particle velocity according to equation (5):

$$
\mathbf{v}_{i}(t+1)=\mathbf{v}_{i}(t)+c_{1}\left(\mathbf{p}_{i}-\mathbf{x}_{i}(t)\right) \mathbf{R}_{1}+c_{2}\left(\mathbf{g}-\mathbf{x}_{i}(t)\right) \mathbf{R}_{2}
$$

b. Update the particle position according to equation (4):

$$
\mathbf{x}_{i}(t+1)=\mathbf{x}_{i}(t)+\mathbf{v}_{i}(t+1)
$$

c. Evaluate the fitness of the particle $f\left(x_{(}(t+1)\right)$.

d. If $f\left(\mathbf{x}_{i}(t+1)\right) \geq f\left(\mathbf{p}_{i}\right)$, update personal best: $\mathbf{p}_{i}=\mathbf{x}_{i}(t+1)$

c. If $f\left(\mathbf{x}_{i}(t+1)\right) \geq f(\mathrm{~g})$, update global best: $\mathbf{g}-\mathbf{x}_{(}(t+1)$

3. At the end of the iterative process, the best solution is represented by g.

Fig. 5. Pseudo code of PSO [29]

\section{Simulation Result}

Performance of Particle Swarm Optimization is analyzed for the purpose of maximizing the achievable rate in beam forming with increasing SNR conditions where SNR is defined as $\rho / \sigma^{\wedge} 2$.

We have compared results of our method with two standard benchmarks of this domain:

Beamforming scheme [17] with continuous angles. It is the best achievable performance possible and we aim to bring our results as close to this as possible. 
Turbo-Like Beamforming [20] based on Tabu Search Algorithm. It uses Tabu search to optimize the pair of analog precoder and combiner.

We consider the antennas at transmitter and receiver ends to be Uniform Linear Arrays (ULAs) with antenna spacing d equal to $\pi / 2$. Carrier frequency is set to $28 \mathrm{GHz}$ and the number of scattering propagation paths (L) is kept 3 . Total number of RF chains at transmission side is the same as the number of RF chains at receiver end, which is in turn equal to the total number of data streams transmitted- which is 2 in our case.

$$
N_{t}^{R F}=N_{r}^{R F}=N_{s}=2
$$

It is assumed that AoAs/AoDs for generating channel matrix from [24] follow uniform distribution in the range $[0, \pi]$. Standard deviation of azimuth and elevation angles of both Rx and Tx is kept to be $10 / 180^{*} \pi$. The complex gain $\alpha$ l of the lth path follows $\alpha \mathrm{l} \sim \mathrm{CN}(0,1)$. Wavelength of transmitted waves is kept to be $2 \mathrm{~mm}$. Particle swarm optimization is used to select such a pair of $P_{A}$ matrix (chosen from precoder codebook F) and $C_{A}$ matrix (chosen from combiner codebook $\mathrm{W}$ ) that maximizes the achievable rate against an SNR range. PSO parameters were set as mentioned in the table below.

Table 1. Parameters for PSO Algorithm

\begin{tabular}{|l|c|}
\hline \multicolumn{1}{|c|}{ PSO Parameters } & Values \\
\hline Number of iterations & 100 \\
\hline Population size & 30 \\
\hline Inertia weight & 1 \\
\hline Damping ratio of inertia weight & 0.99 \\
\hline Learning coefficient- Personal (c1) & 1.5 \\
\hline Learning coefficient- Global (c2) & 2 \\
\hline
\end{tabular}

Experiments were conducted in two different scenarios. In the first one, $\mathrm{Nr}$ was kept to be 16 and Nt was 64. In the second scenario, $\mathrm{Nr}$ was kept to be 32 and Nt was 128 . All other parameters of system model and PSO were kept constant as described above in this section. Results of both these cases were obtained for three different sets of quantified bits per AoAs/AoDs which are:

$$
\begin{aligned}
& B_{t}^{R F}=B_{r}^{R F}=4 \\
& B_{t}^{R F}=B_{r}^{R F}=5 \\
& B_{t}^{R F}=B_{r}^{R F}=6
\end{aligned}
$$


In the figure below are presented the results for the case when $\mathrm{Nr} \times \mathrm{Nt}$ was $16 \times 64$. we can see that for each of the sets of considered quantified bits per AoAs, the results by PSO far exceed those of Turbo TS scheme and approach very closely to the performance by beam steering.

For all considered SNR values, the performance of $B_{t}^{R F}=B_{r}^{R F}=6$ was nearly the same as the performance by beam steering and for higher SNR values, $B_{t}^{R F}=B_{r}^{R F}=$ 5 approached beam steering benchmark as well. The performance at $B_{t}^{R F}=B_{r}^{R F}=4$ was off by the beam steering benchmark by about $1.5 \mathrm{bps} / \mathrm{Hz}$ but it was much higher than the performance of turbo TS method by nearly $3 \mathrm{bps} / \mathrm{Hz}$.

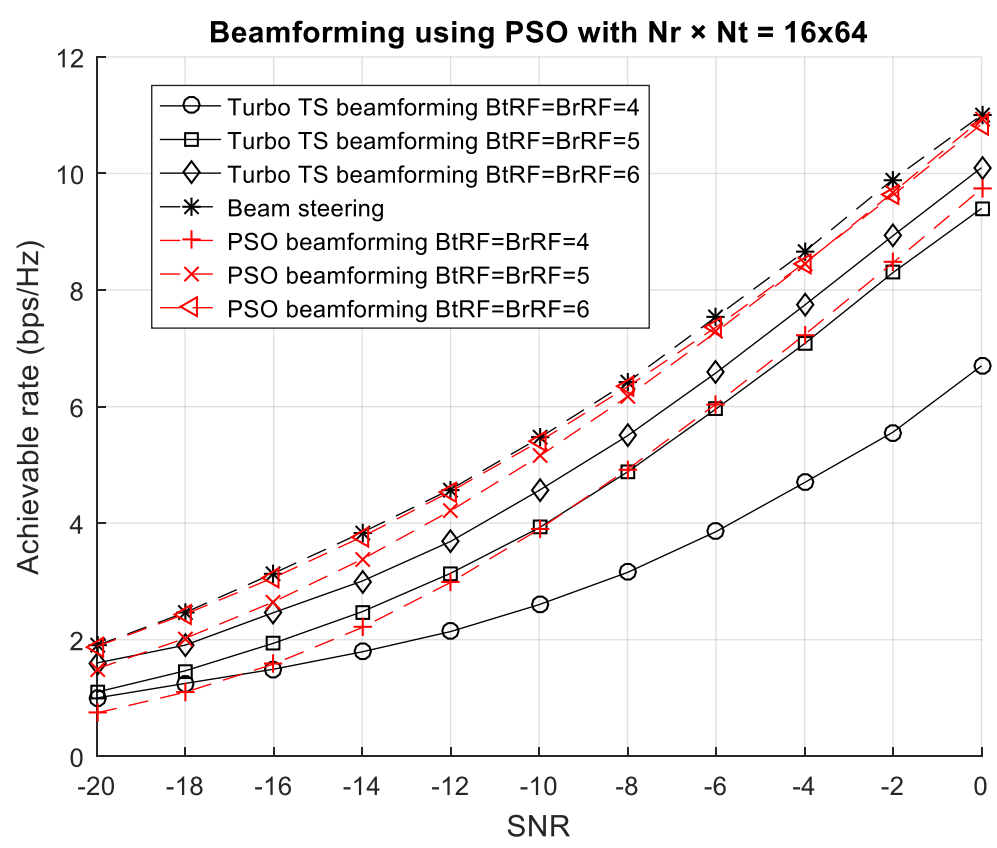

Fig. 6. Beamforming Using PSO with $\mathrm{Nr}^{*} \mathrm{Nt}=16 * 64$

The figure below shows the performance of the proposed system at $\mathrm{N}_{\mathrm{r}} \mathrm{X} \mathrm{N}_{\mathrm{t}}=32 \mathrm{X}$ 128. It can be observed that for $B_{t}^{R F}=B_{r}^{R F}=4$, achievable rate was higher than turbo TS for all values except the starting and ending values. For the other two cases of $B_{t}^{R F}$ $=B_{r}^{R F}=5$ and $B_{t}^{R F}=B_{r}^{R F}=6$ achievable rate was lesser than that of turbo TS in the initial few values of SNR, but after that it becomes better than Turbo TS by a adequate margin, thus mimicking the performance of the beam steering benchmark more closely. 


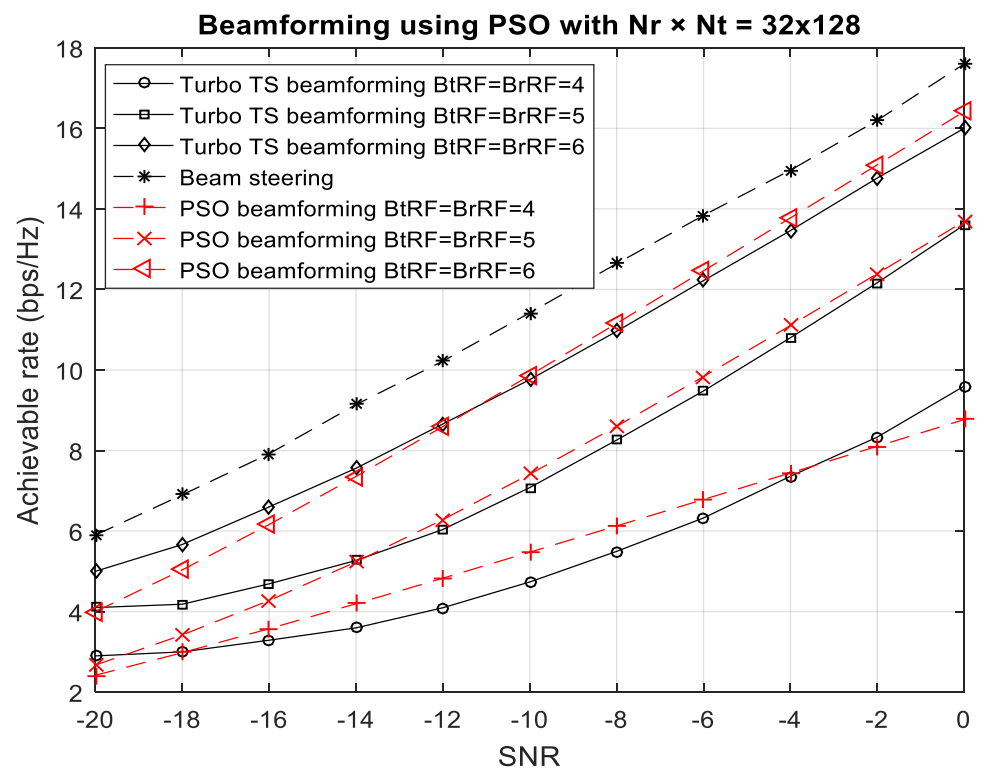

Fig. 7. Beamforming Using PSO with $\mathrm{Nr} * \mathrm{Nt}=32 * 128$

The general trend for both the cases of $\mathrm{N}_{\mathrm{r}} \times \mathrm{N}_{\mathrm{t}}$ is that as the number of quantified bits per AoAs and AoDs ( $B_{t}^{R F}$ and $B_{r}^{R F}$ ) is increased, performance improves and both TurboTS beamforming and PSO based beamforming can achieve results which are much closer to the beam steering benchmark. As a whole, we can say that for majority of the cases, the performance of PSO outperforms that of Turbo TS optimization scheme.

Comparing the above two figures, we can conclude that the performance of the proposed method can be improved by increasing the number of low-cost antennas rather than by increasing the number of expensive RF chains.

\section{Conclusion}

In this work, Particle Swarm Optimization is assessed for the purpose of choosing such a pair of analog precoder and combiner that gives highest achievable rate in $\mathrm{mm}$ wave channel for massive MIMO. Each possible combination of a precoder and a combiner matrix is a particle in the swarm. This optimization technique has fewer parameters to tune and is fast to arrive at a solution regardless of the initial solutions provided to it.

First of all, massive MIMO system was modelled and Saleh-Valenzuela channel was simulated for transmission. Next, a pair of codebooks was generated for analog precoder and combiner. Each codebook had a list of all possible precoder/ combiner matrices. PSO was used to select such indices of precoder and combiner matrices in the 
codebooks that maximize achievable rate upon being paired. Results were simulated over a wide range of SNR from -20 to $0 \mathrm{db}$. It was observed that results by PSO approached those by the beam steering benchmark much more closely than those by Turbo TS optimization [20].

This work is based on ULAs in a single user setup. Future work can check this methodology on UPAs (uniform planar arrays) systems [21] and also on multiple user systems. It can also be modelled for different channels of Massive MIMO [30, 31].

\section{References}

[1] A. Osseiran et al., "Scenarios for 5G mobile and wireless communications: the vision of the METIS project," vol. 52, no. 5, pp. 26-35, 2014.

[2] H. T. Alrikabi, A. H. M. Alaidi, A. S. Abdalrada, and F. T. J. I. J. o. E. T. i. L. Abed, "Analysis the Efficient Energy Prediction for 5G Wireless Communication Technologies," vol. 14, no. 08, pp. 23-37, 2019. https://doi.org/10.3991/ijet.v14i08.10485

[3] J. G. Andrews et al., "What will 5G be?" vol. 32, no. 6, pp. 1065-1082, 2014.

[4] J. Hoydis, S. Ten Brink, and M. J. I. J. o. s. A. i. C. Debbah, "Massive MIMO in the UL/DL of cellular networks: How many antennas do we need?" vol. 31, no. 2, pp. 160-171, 2013. https://doi.org/10.1109/jsac.2013.130205

[5] H. T. S. Al-Rikabi, Enhancement of the MIMO-OFDM Technologies. California State University, Fullerton, 2013.

[6] O. H. Yahya, H. Alrikabi, I. A. J. I. J. o. O. Aljazaery, and B. Engineering, "Reducing the Data Rate in Internet of Things Applications by Using Wireless Sensor Network," vol. 16, no. 03, pp. 107-116, 2020. https://doi.org/10.3991/ijoe.v16i03.13021

[7] K. Doppler, M. Rinne, C. Wijting, C. B. Ribeiro, and K. J. I. C. M. Hugl, "Device-to-device communication as an underlay to LTE-advanced networks," vol. 47, no. 12, pp. 42-49, 2009. https://doi.org/10.1109/mcom.2009.5350367

[8] C. Li, J. Zhang, and K. B. J. I. T. o. W. C. Letaief, "Throughput and energy efficiency analysis of small cell networks with multi-antenna base stations," vol. 13, no. 5, pp. 25052517, 2014. https://doi.org/10.1109/twc.2014.031714.131020

[9] G. Bartoli et al., "Beamforming for small cell deployment in LTE-advanced and beyond," vol. 21, no. 2, pp. 50-56, 2014.

[10] Y. Shi, J. Zhang, K. B. Letaief, B. Bai, and W. J. I. W. C. Chen, "Large-scale convex optimization for ultra-dense cloud-RAN," vol. 22, no. 3, pp. 84-91, 2015. https://doi.org/10.1109/mwc.2015.7143330

[11] T. S. Rappaport et al., "Overview of millimeter wave communications for fifth-generation (5G) wireless networks-With a focus on propagation models," vol. 65, no. 12, pp. 62136230, 2017. https://doi.org/10.1109/tap.2017.2734243

[12] M. R. Akdeniz et al., "Millimeter wave channel modeling and cellular capacity evaluation," vol. 32, no. 6, pp. 1164-1179, 2014

[13] H. T. S. J. W. J. o. E. S. ALRikabi, "Study the Matching of the Level of Electromagnetic Radiation Emitted by Communication Towers in the Kut City with the International Health organization criterion," vol. 4, no. 1, pp. 101-111, 2016.

[14] E. Torkildson, U. Madhow, and M. J. I. T. o. W. C. Rodwell, "Indoor millimeter wave MIMO: Feasibility and performance," vol. 10, no. 12, pp. 4150-4160, 2011. https://doi.org/10.1109/twc.2011.092911.101843 
[15] S. Hur, T. Kim, D. J. Love, J. V. Krogmeier, T. A. Thomas, and A. J. I. t. o. c. Ghosh, "Millimeter wave beamforming for wireless backhaul and access in small cell networks," vol. 61, no. 10, pp. 4391-4403, 2013. https://doi.org/10.1109/tcomm.2013.090513.120848

[16] T. S. Rappaport, R. W. Heath Jr, R. C. Daniels, and J. N. Murdock, Millimeter wave wireless communications. Pearson Education, 2015.

[17] F. Rusek et al., "Scaling up MIMO: Opportunities and challenges with very large arrays," vol. 30, no. 1, pp. 40-60, 2012.

[18] X. Gao, L. Dai, and A. M. J. I. C. M. Sayeed, "Low RF-complexity technologies to enable millimeter-wave MIMO with large antenna array for $5 \mathrm{G}$ wireless communications," vol. 56, no. 4, pp. 211-217, 2018. https://doi.org/10.1109/mcom.2018.1600727

[19] O. El Ayach, R. W. Heath, S. Abu-Surra, S. Rajagopal, and Z. Pi, "The capacity optimality of beam steering in large millimeter wave MIMO systems," in 2012 IEEE 13th International Workshop on Signal Processing Advances in Wireless Communications (SPAWC), 2012, pp. 100-104: IEEE. https://doi.org/10.1109/spawc.2012.6292865

[20] H. T. S. J. I. J. o. S. E. ALRikabi and Research, "Implementation and Estimation of Wireless Communication Channel," vol. 4, no. 10, p. 4, 2016.

[21] H. Alrikabi, A. H. Alaidi, and K. J. I. J. o. I. M. T. Nasser, "The Application of Wireless Communication in IOT for Saving Electrical Energy," vol. 14, no. 01, pp. 152-160, 2020. https://doi.org/10.3991/ijim.v14i01.11538

[22] I. A. Ibrahim, H. T. Salim, and H. F. J. W. J. o. E. S. Khazaal, "Investigating the Analysis of Power Saving Mode in IEEE 802.11 for Wi-Fi Communication," vol. 6, no. 3, pp. 13-19, 2018. https://doi.org/10.31185/ejuow.vol6.iss3.100

[23] X. Gao, L. Dai, C. Yuen, and Z. J. I. T. o. V. T. Wang, "Turbo-like beamforming based on tabu search algorithm for millimeter-wave massive MIMO systems," vol. 65, no. 7, pp. 5731-5737, 2015. https://doi.org/10.1109/tvt.2015.2461440

[24] X. Yu, J.-C. Shen, J. Zhang, and K. B. J. I. J. o. S. T. i. S. P. Letaief, "Alternating minimization algorithms for hybrid precoding in millimeter wave MIMO systems," vol. 10, no. 3, pp. 485-500, 2016. https://doi.org/10.1109/jstsp.2016.2523903

[25] W. Roh et al., "Millimeter-wave beamforming as an enabling technology for 5G cellular communications: Theoretical feasibility and prototype results," vol. 52, no. 2, pp. 106-113, 2014. https://doi.org/10.1109/mcom.2014.6736750

[26] Z. Pi and F. J. I. c. m. Khan, "An introduction to millimeter-wave mobile broadband systems," vol. 49, no. 6, pp. 101-107, 2011.

[27] O. El Ayach, S. Rajagopal, S. Abu-Surra, Z. Pi, and R. W. J. I. t. o. w. c. Heath, "Spatially sparse precoding in millimeter wave MIMO systems," vol. 13, no. 3, pp. 1499-1513, 2014. https://doi.org/10.1109/twc.2014.011714.130846

[28] T. Kim, J. Park, J.-Y. Seol, S. Jeong, J. Cho, and W. Roh, "Tens of Gbps support with mmWave beamforming systems for next generation communications," in 2013 IEEE Global Communications Conference (GLOBECOM), 2013, pp. 3685-3690: IEEE. https://doi.org/10.1109/glocom.2013.6831646

[29] X.-S. Yang, Engineering optimization: an introduction with metaheuristic applications. John Wiley \& Sons, 2010.

[30] A. H. Gandomi, X.-S. Yang, S. Talatahari, and A. H. Alavi, Metaheuristic applications in structures and infrastructures. Newnes, 2013. https://doi.org/10.1016/b978-0-12-3983640.00001-2

[31] F. Marini, B. J. C. Walczak, and I. L. Systems, "Particle swarm optimization (PSO). A tutorial," vol. 149, pp. 153-165, 2015. https://doi.org/10.1016/j.chemolab.2015.08.020 
[32] E. G. Larsson, O. Edfors, F. Tufvesson, and T. L. J. I. c. m. Marzetta, "Massive MIMO for next generation wireless systems," vol. 52, no. 2, pp. 186-195, 2014. https://doi.org/10.1109/mcom.2014.6736761

[33] L. You, X. Gao, X.-G. Xia, N. Ma, and Y. J. I. T. o. W. C. Peng, "Pilot reuse for massive MIMO transmission over spatially correlated Rayleigh fading channels," vol. 14, no. 6, pp. 3352-3366, 2015. https://doi.org/10.1109/twc.2015.2404839

\section{$7 \quad$ Authors}

Thaar A. Kareem is an assistant lecturer in Electric Engineering College of Engineering, University of Misan, Iraq. He obtained BSC from university of Baghdad, Iraq and the master from EMU, north Cyprus. E-mail: thaar kareem@uomisan.edu.iq.

Maab Alaa Hussain is an assistant lecturer in Civil Engineering College of Engineering, university of Misan, Iraq. She obtained BSC from university of Baghdad, Iraq and the master from university of Baghdad. E-mail: maab alaa@uomisan.edu.iq.

Mays Kareem Jabbar is a lecturer in Electric Engineering College of Engineering, university of Misan, Iraq. She obtained BSC from university of technology, Iraq and the master from EMU, north Cyprus. E-mail: $\mathrm{m}$ mays85@uomisan.edu.iq.

Article submitted 2020-02-11. Resubmitted 2020-03-03. Final acceptance 2020-03-17. Final version published as submitted by the authors. 